PHYSICAL REVIEW D 84, 063523 (2011)

\title{
Gravitational waves in the presence of a cosmological constant
}

\author{
José Bernabeu \\ Departament de Física Teòrica IFIC, Universitat de València-CSIC, Dr. Moliner 50, 46100 Burjassot, València, Spain \\ Domènec Espriu and Daniel Puigdomènech \\ Departament d'Estructura i Constituents de la Matèria, Institut de Ciències del Cosmos (ICCUB) Universitat de Barcelona, \\ Martí i Franquès, 1, 08028 Barcelona, Spain
}

(Received 23 June 2011; published 26 September 2011)

\begin{abstract}
We derive the effects of a nonzero cosmological constant $\Lambda$ on gravitational wave propagation in the linearized approximation of general relativity. In this approximation, we consider the situation where the metric can be written as $g_{\mu \nu}=\eta_{\mu \nu}+h_{\mu \nu}^{\Lambda}+h_{\mu \nu}^{W}, h_{\mu \nu}^{\Lambda, W} \ll 1$, where $h_{\mu \nu}^{\Lambda}$ is the background perturbation and $h_{\mu \nu}^{W}$ is a modification interpretable as a gravitational wave. For $\Lambda \neq 0$, this linearization of Einstein equations is self-consistent only in certain coordinate systems. The cosmological Friedmann-RobertsonWalker coordinates do not belong to this class and the derived linearized solutions have to be reinterpreted in a coordinate system that is homogeneous and isotropic to make contact with observations. Plane waves in the linear theory acquire modifications of order $\sqrt{\Lambda}$, both in the amplitude and the phase, when considered in Friedmann-Robertson-Walker coordinates. In the linearization process for $h_{\mu \nu}$, we have also included terms of order $\mathcal{O}\left(\Lambda h_{\mu \nu}\right)$. For the background perturbation $h_{\mu \nu}^{\Lambda}$, the difference is very small, but when the term $h_{\mu \nu}^{W} \Lambda$ is retained the equations of motion can be interpreted as describing massive spin-2 particles. However, the extra degrees of freedom can be approximately gauged away, coupling to matter sources with a strength proportional to the cosmological constant itself. Finally, we discuss the viability of detecting the modifications caused by the cosmological constant on the amplitude and phase of gravitational waves. In some cases, the distortion with respect to gravitational waves propagating in Minkowski space-time is considerable. The effect of $\Lambda$ could have a detectable impact on pulsar timing arrays.
\end{abstract}

DOI: 10.1103/PhysRevD.84.063523

PACS numbers: 04.30.- w, 98.80.Es

\section{INTRODUCTION}

The smallness of the cosmological constant obtained from fits to the current $\Lambda \mathrm{CDM}$ cosmological models [1] $\left(\Lambda \simeq 10^{-52} \mathrm{~m}^{-2}\right)$ may lead us to believe that it is totally unobservable except at the largest distances. However, the issue of the relevance of the cosmological constant in local measurements (meaning measurements that involve subcosmological scales, such as with galaxy clusters) has received growing attention [2,3]. One interesting possibility is assessing the influence of $\Lambda$ on the bending of light from distant objects. At present there are rather diverging results on the subject giving rather different results concerning the relevance of $\Lambda$ ranging from zero [4] or very small [5] to appreciable ones [6]. The effect of $\Lambda$ on the photon propagation, including frequency shift, Shapiro time delay, and deflection of light, is currently under consideration [7].

The importance of these studies cannot be overemphasized. The presence of a nonzero cosmological constant contributing around $70 \%$ to the energy and matter budget of the Universe, seemingly making the Universe globally a de Sitter space-time, is one of the intriguing puzzles of physics in our time. Observations capable of confirming or refuting the relevance of $\Lambda$ at redshift $z<1$ are clearly of the utmost importance.
The studies of what has been termed "local gravity with a cosmological constant" rely on an approximate solution, valid at first order in $\Lambda$, obtained after linearizing Einstein equations. These solutions have recently been studied in detail by one of the authors [3] using different gauge choices. It has been found that in the Lorenz gauge one can in addition require time independence of the metric solutions. After an additional coordinate transformation, these solutions correspond to the linearized version of the Schwarzschild-de Sitter exact solution of Einstein equations. The modification to the Newtonian limit in such coordinates was also discussed in detail [3]. There are some subtleties related to the physical interpretation of the different coordinate systems that we shall review below.

Here we propose to study a different problem. Namely, how $\Lambda$ influences the properties of gravitational waves (GW). As of today, gravitational waves are an unambiguous prediction of general relativity that has not been tested directly. They are "observed" indirectly as they are the missing ingredient needed to restore the energy balance of some astrophysical binary systems [8]. There are three types of experiments potentially capable of yielding a nonzero signal in the coming years. Let us summarize their physical and astrophysical reach here: 
Ground-based GW detectors such as LIGO [9] can reach sensitivities down to $\sim 10^{-23}$ with optimal sensitivity in the region between $10 \mathrm{~Hz}$ and $10^{3} \mathrm{~Hz}$. The space mission LISA [10] will reach a similar sensitivity in the range $10^{-2} \mathrm{~Hz}$ to $10^{-3} \mathrm{~Hz}$ but will actually be able to set relevant bounds on a more extended range of frequencies. Finally, the International Pulsar Timing Array project [11] or the Square Kilometer Array project [12] are sensitive to lower frequencies $\nu<10^{-4} \mathrm{~Hz}$ but reach only a sensitivity of $\sim 10^{-10}$ going up to $\sim 10^{-15}$ for $\nu \sim 10^{-10} \mathrm{~Hz}$. These sensitivity ranges are targeted to specific astrophysical phenomena and are expected to provide detectable signals and confirm the existence of GW in the coming decades.

Given the present difficulties in asserting the very existence of GW, it may seem academic to try to find modifications due to the presence of a cosmological constant that is small. However, it should be borne in mind that in the inflationary epoch the value of $\Lambda$ was much larger than at present, so these effects might be of relevance for primordial GW. As we will discuss in this work, the effect of $\Lambda$ could be of some relevance for GW traveling very long distances and for pulsar timing array projects. On the other hand, some of the results presented here we believe are of interest to understand the issue of the gauge choice in the presence of $\Lambda$ for the linear theory. Finally, it seems interesting in its own right to attempt to understand wave propagation in de Sitter space-time if $\Lambda$ is indeed a fundamental parameter of nature. Our approach corresponds to a "local gravity" discussion, assuming, even in the presence of $\Lambda$, the gravitational field as a linear perturbation around Minkowski's space-time. This expansion applies to a region of distances smaller than the de Sitter horizon $l \ll \sqrt{\frac{3}{\Lambda}}$. We will argue that this linearization is consistent in some coordinate systems but not in others.

This paper is organized as follows. In Sec. II, we discuss the linearization of Einstein equations, including a discussion on different gauges and how they affect the wave equation for the gravitational field $h_{\mu \nu}$. In Sec. III, we discuss different coordinate realizations of de Sitter space-time and their relation. In Sec. IV, we construct background solutions retaining terms of order $\Lambda h_{\mu \nu}$. This discussion is extended in Sec. V to include GW solutions that "feel" the presence of $\Lambda$. In Sec. VI, we analyze the detectability of the effects previously calculated. In Sec. VII, we summarize the conclusions of this study.

Some of the subjects discussed here appear to have received little attention in the past although there is extensive literature on gravitational waves [13]. The effect of $\Lambda$ on GW has been considered in $[14,15]$ and, in certain gauges, linear perturbations on the Friedmann-RobertsonWalker (FRW) backgrounds have been considered [16]. Physical consequences appear to have been extracted in the context of primordial gravitational waves [17] and only indirectly in what concerns the evolution of the modes and the power spectrum.

\section{LINEARIZATION IN THE PRESENCE OF $\Lambda$}

Einstein equations, derived from the Einstein-Hilbert action, read

$$
R_{\mu \nu}-\frac{1}{2} g_{\mu \nu} R+\Lambda g_{\mu \nu}=-\kappa T_{\mu \nu}
$$

where $R_{\mu \nu}$ is the Ricci tensor for $g_{\mu \nu}, \Lambda>0$ is the cosmological constant, and $\kappa T_{\mu \nu}$ is the source term. $T_{\mu \nu}$ is the usual stress-energy tensor of matter in the gravitational field generated by $g_{\mu \nu}$ and $\kappa$ is the dimensionful constant coupling matter and gravity. However, throughout this work we will consider $T_{\mu \nu}=0$ unless otherwise specified. The inclusion of the cosmological constant term leads to curvature even in the absence of any source

$$
R=4 \Lambda \text {. }
$$

We consider the linearized theory where the metric is written as

$$
g_{\mu \nu}=\eta_{\mu \nu}+h_{\mu \nu},
$$

$\eta_{\mu \nu}$ being the Minkowski metric and $h_{\mu \nu} \ll 1$. The Ricci tensor to first order in the small perturbation $h_{\mu \nu}$ reads

$$
R_{\mu \nu}=\frac{1}{2}\left(\square h_{\mu \nu}+h_{, \mu \nu}-h_{\mu, \nu \lambda}^{\lambda}-h_{\nu, \mu \lambda}^{\lambda}\right),
$$

indices being lowered and raised with $\eta_{\mu \nu}$ and $h=$ $\eta^{\mu \nu} h_{\mu \nu}$. The theory is invariant under coordinate transformations $x^{\mu} \rightarrow x^{\mu}+\xi^{\mu}(x)$. For infinitesimal transformations, the perturbation metric $h_{\mu \nu}$ transforms as $h_{\mu \nu} \rightarrow h_{\mu \nu}^{\prime}=h_{\mu \nu}+\partial_{\mu} \xi_{\nu}+\partial_{\nu} \xi_{\mu}$. A gauge choice is possible, amounting to selecting a particular class of coordinates, and in fact such a choice is necessary if the perturbation $h_{\mu \nu}$ is to be quantized. In order to discuss GW, two different gauge choices are particularly appropriate.

\section{A. Lorenz gauge}

In order to describe perturbations around flat space-time, it is customary to employ the Lorenz gauge.

$$
\partial_{\mu} h_{\nu}^{\mu}=\frac{1}{2} \partial_{\nu} h,
$$

or

$$
\partial_{\mu} \tilde{h}_{\nu}^{\mu}=0,
$$

where

$$
\tilde{h}_{\mu \nu}=h_{\mu \nu}-\frac{1}{2} \eta_{\mu \nu} h
$$

is the trace reversed version of $h_{\mu \nu}$.

In this gauge, expression (4) is simplified to

$$
R_{\mu \nu}=\frac{1}{2} h_{\mu \nu},
$$


and we obtain the equation of motion

$$
\square\left(h_{\mu \nu}-\frac{1}{2} \eta_{\mu \nu} h\right)+2 \Lambda h_{\mu \nu}=-2 \Lambda \eta_{\mu \nu},
$$

which must always be considered together with the Lorenz gauge condition (5).

Whether the term of order $\mathcal{O}(h \Lambda)$ has to be considered depends on the relative magnitude of $h$ and $\Lambda$. There will be situations when the inclusion of this term is justified and may lead to observable consequences. We shall postpone the rest of the discussion on this issue until sections IV and V. Note, nonetheless, that if the $\Lambda h_{\mu \nu}$ term on the left-hand side is omitted (and only in this case), there is a residual gauge freedom within the Lorenz gauge. If we perform a linear coordinate transformation

$$
x^{\mu} \rightarrow x^{\prime \mu}=x^{\mu}+\xi^{\mu},
$$

Equation (5) is fulfilled as long as $\xi^{\mu}$ is an harmonic function, i.e., $\square \xi^{\mu}=0$. These residual coordinate transformations are sometimes termed "coordinate waves" for rather obvious reasons. Note also that whether this is a symmetry of the equations of motion depends on the terms retained in the linearization; the term $\Lambda h_{\mu \nu}$ breaks this residual coordinate invariance.

\section{B. $\Lambda$ gauge}

It will be useful to consider an alternative gauge choice [18], which we will term $\Lambda$ gauge. This is given by the gauge condition

$$
\partial_{\mu} \tilde{h}_{\nu}^{\mu}=-\Lambda \eta_{\nu \mu} x^{\mu}
$$

In this gauge, the linearized equations of motion look slightly different

$$
\square\left(h_{\mu \nu}-\frac{1}{2} \eta_{\mu \nu} h\right)-2 \Lambda h_{\mu \nu}=0 .
$$

In particular, we note that the term independent of $h_{\mu \nu}$ on the right-hand side of (9) is absent. There is a set of coordinate transformations that can be performed without leaving the gauge orbit (11); these are transformations $x^{\prime \mu}=x^{\mu}+\xi^{\mu}$ with

$$
\square \xi^{\mu}=-\Lambda \xi^{\mu} .
$$

However, in the $\Lambda$ gauge these residual coordinate transformations are not a symmetry of the equations of motion regardless of the terms retained in the linearization and therefore cannot be used to remove degrees of freedom. Generally speaking, linearization leaves global Lorentz transformations as the only symmetry of the equations of motion. The Lorenz gauge is in a way special as some additional freedom to perform local coordinate transformations remains if the term $\Lambda h_{\mu \nu}$ is neglected. The situation in the $\Lambda$ gauge, where there is no residual symmetry, is, on the contrary, the generic one.

The connection between the two gauge choices in the linear theory is easily made when the terms $\Lambda h_{\mu \nu}$ are omitted. It is implemented via the following change of coordinates

$$
x^{\mu} \rightarrow x^{\prime \mu}=x^{\mu}+\xi^{\mu}=\left(1-\frac{\Lambda}{12} x^{2}\right) x^{\mu} .
$$

This change of coordinates transforms a solution of $\square \tilde{h}_{\mu \nu}=0$ in the $\Lambda$ gauge (coordinates $x$ ) to a solution of $\square \tilde{h}_{\mu \nu}=-2 \Lambda \eta_{\mu \nu}$ in Lorenz gauge (coordinates $x^{\prime}$ ). Note the simplicity of the equation for linear perturbations in the $\Lambda$ gauge if the term of order $\Lambda h_{\mu \nu}$ is omitted. All reference to the cosmological constant is eliminated.

The previous discussion of the $\Lambda$ gauge reminds us that in general, in the linearized approximation, the perturbation metric $h_{\mu \nu}$ is expected to have up to six full degrees of freedom. Only in certain cases a residual gauge freedom can be used to further reduce the number of degrees of freedom.

Let us elaborate a bit more on this issue as it is conceptually important. In the Lorenz gauge, with the term $\Lambda h_{\mu \nu}$ omitted, the residual symmetry (10) allows us to move freely between different coordinate systems, say $x^{\prime}$ and $y^{\prime}$, which are not trivially related by Lorentz transformations and yet preserve the form of the equations of motion. On the contrary, if we undo transformation (14) we get two coordinate systems $x$ and $y$ in which the $\Lambda$ gauge condition is fulfilled but at best only one of these gauge-transformed coordinate systems obeys the linearized equations of motion in the $\Lambda$ gauge; the other one is off shell. That is to say, the number of independent degrees of freedom seems to be larger in the $\Lambda$ gauge. However, since this is purely due to a gauge choice, the additional apparent degrees of freedom cannot correspond to physical ones.

If the term of order $\Lambda h_{\mu \nu}$ is retained, i.e., in the Lorenz gauge the term $2 \Lambda h_{\mu \nu}$ on the left-hand side of (9) or the analogous $-2 \Lambda h_{\mu \nu}$ in the $\Lambda$ gauge are kept, there is no residual symmetry whatsoever. Let us take for example (9) in the Lorenz gauge; as we will see in detail in Sec. V, this generates a genuine mass term and therefore more physical degrees of freedom appear associated to $h_{\mu \nu}$. This is not a gauge artifact.

\section{DE SITTER SPACE-TIME}

De Sitter space-time can be described by many coordinate systems. A convenient choice of coordinates is Schwarzschild-de Sitter (SdS). These provide a timeindependent metric in a gauge that is none of the two previously discussed

$d s^{2}=\left[1-\frac{\Lambda}{3} \hat{r}^{2}\right] d \hat{t}^{2}-\left[1-\frac{\Lambda}{3} \hat{r}^{2}\right]^{-1} \hat{r}^{2}+\hat{r}^{2} d \Omega^{2}$ 
and clearly shows the presence of the de Sitter horizon. We note that this metric admits an expansion in integer powers of $\Lambda$. Note also that in this metric the spatial part does not quite correspond to spherical coordinates.

At the opposite extreme, one can select a metric that depends only on time and is position independent. It is the Friedmann-Robertson-Walker (FRW) metric

$$
d s^{2}=d T^{2}-\exp \left(2 \sqrt{\frac{\Lambda}{3}} T\right) d \vec{X}^{2}
$$

This metric incorporates the physical principles of cosmological homogeneity and isotropy as it does not depend on the position. The coordinates $X^{i}$ have a clear physical meaning: they are comoving coordinates anchored in space that expand with the universe. These are the natural coordinates where our world appears homogeneous and isotropic. It is easy to see that the FRW metric does not fulfill any linearized Einstein equation, even for very early times $t \ll 1 / \sqrt{\Lambda}$ when it is very close to the Minkowski metric. In fact, no metric that depends only on time can be a solution of the linearized Einstein equations; incompatibilities appear immediately for any gauge choice.

One should therefore accept that the linearized Einstein equations in the presence of $\Lambda$ cannot be imposed in the physically relevant comoving coordinate system. This, of course, has implications on GW as the very concept of "wave" does require a wave equation, which is just impossible in FRW coordinates. On the other hand, the wave equation $\square \tilde{h}_{\mu \nu}=0$ found in the $\Lambda$ gauge is expressed in a set of coordinates whose meaning is yet to be interpreted. Therefore, the simplicity of this equation is deceiving.

We will argue in the next section that the coordinates implied by the choice of the $\Lambda$ gauge or of the Lorenz gauge are closely related to $\mathrm{SdS}$ coordinates. Then the way to proceed is to find a solution for GW in the Lorenz gauge, a coordinate system where linearization of the Einstein equations is consistent, and then transform the solution to FRW coordinates in order to extract observable consequences.

Both the SdS metric and the FRW metric are valid (but rather different) descriptions of de Sitter geometry. One can work out the exact transformation between the two coordinate systems

$\hat{r}=e^{T \sqrt{\Lambda / 3}} R \quad \hat{t}=\sqrt{\frac{3}{\Lambda}} \log \left(\frac{\sqrt{3}}{\sqrt{3-\Lambda e^{2 T \sqrt{\Lambda / 3}} R^{2}}}\right)+T$,

where $T$ and $R$ are, respectively, the cosmological time and comoving coordinates whose physical realization is clear. This transformation is valid inside the cosmological horizon, i.e., $R<\frac{1}{\sqrt{\Lambda}}$. Applying (17) to (15), we obtain

$$
d s^{2}=d T^{2}-\exp \left(2 \sqrt{\frac{\Lambda}{3}} T\right) d \vec{X}^{2} .
$$

Now it is immediate to see that the FRW metric does not fulfill any linearized Einstein equation, even if $t \ll 1 / \sqrt{\Lambda}$ as it is not expandable in integer powers of $\Lambda$. The same transformations for the linearized version of the metrics gives

$d s^{2}=\left[1-\frac{\Lambda}{3} \hat{r}^{2}\right] d \hat{t}^{2}-\left[1+\frac{\Lambda}{3} \hat{r}^{2}\right] \hat{r}^{2}+\hat{r}^{2} d \Omega^{2}$
$d s^{2}=d T^{2}-\left[1+2 \sqrt{\frac{\Lambda}{3}} T+2 \frac{\Lambda}{3} T^{2}\right]\left(d R^{2}+R^{2} d \Omega^{2}\right)$,

which will only reasonably approximate the expansion of FRW for values of $R \sim T \ll \frac{1}{\sqrt{\Lambda}}$. Note that, although the last metric in (19) is linearized, it does not fulfill any linearized Einstein equations.

The previous transformation provides the relationship between a framework where the Einstein equations can be consistently linearized and the actual coordinate system in which we observe. The solutions easily found in the linearized theory have to be transformed to the physically meaningful coordinate system in order to make predictions. It is at this point that nontrivial effects related to $\Lambda$ will appear. They are discussed in Sec. V. Of course, given the current value of $\Lambda$, these effects will be small. We believe, nonetheless, that these corrections are conceptually important. Note also that (17) involves $\sqrt{\Lambda}$ and not $\Lambda$, yielding corrections that are potentially much more relevant for observation than those of the order $\mathcal{O}(\Lambda)$.

Equation (16) is just one of the many possible cosmological FRW metrics. Other possibilities such as a powerlaw cosmological scale factor do not correspond to a de Sitter space-time and therefore there is no obvious change of coordinates that allows us to reexpress a $\mathrm{GW}$, i.e., a solution to a wave equation, in that physically meaningful coordinate system.

\section{BACKGROUND SOLUTIONS}

We shall work consistently in the linearized approximation both for the background modification $h_{\mu \nu}^{\Lambda}$ and for gravitational wave perturbations $h_{\mu \nu}^{W}$. Namely, the metric can be written as $g_{\mu \nu}=\eta_{\mu \nu}+h_{\mu \nu}^{\Lambda}+h_{\mu \nu}^{W}$, where $h_{\mu \nu}^{\Lambda, W} \ll 1$. To keep the notation simple, we shall only use the superscript $\Lambda$ when confusion with wave perturbations $h_{\mu \nu}^{W}$ is possible. In this section, we will be concerned with background linearized solutions when the cosmological constant $\Lambda$ is present.

The value of the cosmological constant has presumably not been the same throughout the history of the universe. In early epochs, perhaps following an inflationary period, its 
value is believed to have been much larger [19]. This fact suggests that it may be necessary in some circumstances to retain the term $\Lambda h_{\mu \nu}^{\Lambda}$. Likewise, it will be necessary for consistency to keep terms of order $\Lambda h_{\mu \nu}^{W}$ as the magnitudes of $h_{\mu \nu}^{W}$ and $\Lambda$ are unrelated.

In what follows, we proceed without making any assumptions on the value of $\Lambda$; we will just assume that the perturbation that induces on the background metric $h_{\mu \nu}$ is small enough for the linearized approximation to be meaningful.

\section{A. Lowest order solutions}

First, we turn to the lowest order solutions already discussed in [3], which correspond to neglecting terms of $\mathcal{O}\left(\Lambda h_{\mu \nu}\right)$. In the Lorenz gauge, this amounts to solving the following equation,

$$
\square \tilde{h}_{\mu \nu}=-2 \Lambda \eta_{\mu \nu} \quad \partial_{\mu} \tilde{h}_{\nu}^{\mu}=0 .
$$

Linearization limits the validity of the solution to values of the coordinates such that $x^{2} \ll 1 / \Lambda$.

Before discussing the solutions to (20), we take a look at the equations in the $\Lambda$ gauge

$$
\square \tilde{h}_{\mu \nu}=0 \quad \partial_{\mu} \tilde{h}_{\nu}^{\mu}=-\Lambda \eta_{\nu \mu} x^{\mu} .
$$

Note once more that the linearized equations are not invariant under gauge transformations. In the Lorenz gauge, the cosmological constant is regarded as a gravitational source, it appears in the equations of motion, whereas in the $\Lambda$ gauge all dependency in the cosmological constant at this order appears through the gauge condition only and in a way it can be interpreted as a consequence of the coordinate choice. ${ }^{1}$ The connection between the two gauge choices in the linear theory has already been discussed.

We can easily solve Eqs. (21) to find the traceless solution

$$
\tilde{h}_{\mu \nu}=-\frac{\Lambda}{18}\left(4 x_{\mu} x_{\nu}-\eta_{\mu \nu} x^{2}\right) .
$$

If we require that the solution is proportional to $\Lambda$ and involves only the coordinates $x^{\mu}$, this is the unique solution. In addition, (22) is the only one that is Lorentzcovariant (note that $\eta_{\mu \nu}$ is the underlying metric and there is no other four-vector at our disposal).

It is worth noticing that since there is no residual freedom in this gauge, no transformation can turn this solution into a static metric: The $\Lambda$ gauge is explicitly incompatible with the solutions being static.

We now transform the solution back to the Lorenz gauge using (14). We find

\footnotetext{
${ }^{1}$ This, of course, does not mean that the consequences of $\Lambda$ can be removed by a wise coordinate transformation, but it does mean that it disappears from the equations of motion themselves.
}

$$
h_{\mu \nu}=\frac{\Lambda}{9}\left(x_{\mu} x_{\nu}+2 \eta_{\mu \nu} x^{2}\right) .
$$

Without the $\Lambda h_{\mu \nu}$ term, the equation of motion is actually invariant under residual transformations. The number of physical degrees of freedom therefore is reduced to two. This is the only covariant-looking solution in the Lorenz gauge but only one of the infinite number of solutions reachable by noncovariant residual transformations. The most general form of such transformations is

$$
\xi^{\prime \mu}=\left(\begin{array}{c}
A\left(t^{2}+r^{2}\right) t \\
\left(B_{1} t^{2}+B_{2} x^{2}+B_{3}\left(y^{2}+z^{2}\right)\right) x \\
\left(B_{1} t^{2}+B_{2} y^{2}+B_{3}\left(x^{2}+z^{2}\right)\right) y \\
\left(B_{1} t^{2}+B_{2} z^{2}+B_{3}\left(x^{2}+y^{2}\right)\right) z
\end{array}\right),
$$

where $2 B_{1}-6 B_{2}-4 B_{3}=0$. In particular, we find the values of these constants that allow us to reproduce the static solution of [3],

$$
\begin{aligned}
A & =-\frac{\Lambda}{18}, & B_{1} & =-\frac{\Lambda}{9}, \\
B_{2} & =-\frac{\Lambda}{18}, & B_{3} & =\frac{\Lambda}{36} ;
\end{aligned}
$$

one should ask at this point what are these coordinates. We already know that they cannot correspond to cosmological coordinates. In fact, the resulting metric is neither homogeneous nor isotropic although it preserves the symmetry among the three axes. The answer becomes obvious once one discovers that one of the possible residual gauge transformations eliminates the time dependence of the metric. A generalization of Birkhoff's theorem [20] states that there is a unique static solution with spherical symmetry which is the Schwarzschild-de Sitter metric previously discussed, or more precisely the first order of it in the $\Lambda$ expansion. Since Schwarzschild-de Sitter does not fulfill the Lorenz gauge condition, a time-independent coordinate transformation must also be involved. Let us explicitly show this point using a succession of coordinate transformations linear in $\Lambda$.

The first step is to transform (23) to a static solution. We start from

$$
\begin{aligned}
d s^{2}= & {\left[1+\frac{\Lambda}{9}\left(3 t^{2}-2 r^{2}\right)\right] d t^{2} } \\
& -\left[1-\frac{\Lambda}{9}\left(-2 t^{2}+2 r^{2}+x^{i^{2}}\right)\right] d x^{i^{2}} \\
& -\frac{2 \Lambda}{9} t x^{i} d t d x^{i}+\frac{2 \Lambda}{9} x^{i} x^{j} d x^{i} d x^{j},
\end{aligned}
$$

where $i=1,2,3$ and $i \neq j$. After the following change of coordinates, 


$$
\begin{aligned}
& x=x^{\prime}+\frac{\Lambda}{9}\left(-t^{\prime 2}-\frac{x^{\prime 2}}{2}+\frac{\left(y^{\prime 2}+z^{\prime 2}\right)}{4}\right) x^{\prime} \\
& y=y^{\prime}+\frac{\Lambda}{9}\left(-t^{\prime 2}-\frac{y^{\prime 2}}{2}+\frac{\left(x^{\prime 2}+z^{\prime 2}\right)}{4}\right) y^{\prime} \\
& z=z^{\prime}+\frac{\Lambda}{9}\left(-t^{\prime 2}-\frac{z^{\prime 2}}{2}+\frac{\left(x^{\prime 2}+y^{\prime 2}\right)}{4}\right) z^{\prime} \\
& t=t^{\prime}-\frac{\Lambda}{18}\left(t^{\prime 2}+r^{\prime 2}\right) t^{\prime},
\end{aligned}
$$

the metric transforms into the static solution to order $\Lambda$ found in [3],

$d s^{2}=\left[1-\frac{\Lambda}{3} r^{\prime 2}\right] d t^{\prime 2}-\left[1-\frac{\Lambda}{6}\left(r^{\prime 2}+3 x_{i}^{\prime 2}\right)\right] d x_{i}^{\prime 2}$.

Note that this solution is still in the Lorenz gauge; we only performed a residual gauge transformation that is allowed in this gauge. Since our starting solution is only valid to order $\Lambda$, in any change of coordinates, either exact or linear, we only keep terms linear in the cosmological constant. We can further transform (28) to obtain a fully spherically-symmetric solution. Under the following change,

$$
\begin{aligned}
x^{\prime} & =x^{\prime \prime}+\frac{\Lambda}{12} x^{\prime \prime 3} & y^{\prime} & =y^{\prime \prime}+\frac{\Lambda}{12} y^{\prime \prime 3} \\
z^{\prime} & =z^{\prime \prime}+\frac{\Lambda}{12} z^{\prime \prime 3} & t^{\prime} & =t^{\prime \prime},
\end{aligned}
$$

we obtain

$d s^{2}=\left[1-\frac{\Lambda}{3} r^{\prime \prime 2}\right] d t^{\prime \prime 2}-\left[1-\frac{\Lambda}{6} r^{\prime \prime 2}\right]\left(d r^{\prime \prime 2}+r^{\prime \prime 2} d \Omega^{2}\right)$,

which does not obey (20) anymore. We can now perform another coordinate transformation to obtain the SdS metric to order $\Lambda$

$$
\begin{gathered}
r^{\prime \prime}=\hat{r}+\frac{\Lambda}{12} \hat{r}^{3} \quad t^{\prime \prime}=\hat{t} \\
d s^{2}=\left[1-\frac{\Lambda}{3} \hat{r}^{2}\right] d \hat{t}^{2}-\left[1+\frac{\Lambda}{3} \hat{r}^{2}\right] d \hat{r}^{2}+\hat{r}^{2} d \Omega^{2} .
\end{gathered}
$$

This is the linearized Schwarzschild-de Sitter metric. Essentially, the background solution (23) is the $\mathrm{SdS}$ metric in a set of coordinates related to $\mathrm{SdS}$ by timeindependent transformations.

\section{B. Next-order solutions}

Let us now relax the approximation of the previous section and retain terms proportional to $\Lambda h_{\mu \nu}$. In particular, we will be interested later in terms of order $\Lambda h_{\mu \nu}^{W}$ that will influence the propagation of gravitational waves.

In the Lorenz gauge, this requires the simultaneous fulfillment of the two sets of Eqs. (5) and (9). We note that because of the dimensionality of $\Lambda$, any solution of the previous equations containing $\Lambda$ and constructed with the only available (Lorentz-)covariant vector $x^{\mu}$ must necessarily be even under a change of sign of all coordinates $x^{\mu} \rightarrow-x^{\mu}$. Solutions odd in $x^{\mu}$ exist, but they require the involvement of parameters other than the coordinates and $\Lambda$ (a wave vector, for instance; see Sec. IV).

The most general solution of this equation can be written as a superposition of both complex and real exponentials

$$
\begin{aligned}
h_{\mu \nu}= & \int \frac{d^{4} k}{(2 \pi)^{4}} \delta\left(k^{2}-2 \Lambda\right)\left(E_{\mu \nu} \cos k x+D_{\mu \nu} \sin k x\right. \\
& \left.+\frac{\eta_{\mu \nu}}{4}(A \cosh k x+B \sinh k x)\right)-\eta_{\mu \nu},
\end{aligned}
$$

with $E_{\mu \nu}$ and $D_{\mu \nu}$ traceless, i.e., $E_{\mu}^{\mu}=D_{\mu}^{\mu}=0$. In the previous expression, $E_{\mu \nu}, D_{\mu \nu}, A$, and $B$ are in principle all independent functions of $k$ provided that the two following gauge conditions are met,

$$
\int \frac{d^{4} k}{(2 \pi)^{4}} \delta\left(k^{2}-2 \Lambda\right)\left(k_{\mu} E_{\nu}^{\mu} \sin k x+\frac{k_{\nu}}{4} A \sinh k x\right)=0
$$

$$
\int \frac{d^{4} k}{(2 \pi)^{4}} \delta\left(k^{2}-2 \Lambda\right)\left(k_{\mu} D_{\nu}^{\mu} \cos k x-\frac{k_{\nu}}{4} B \cosh k x\right)=0 .
$$

Clearly, the integrands involved have to fall off sufficiently fast for large values of $k$ for the integrals to exist.

This solution has 10 degrees of freedom to start with. Nine come from $E_{\mu \nu}$ and $D_{\mu \nu}$ after removal of the trace. Another one comes from the coefficients $A, B$. Note that both $A$ and $B$ are needed to provide a full degree of freedom and likewise for $E_{\mu \nu}$ and $D_{\mu \nu}$. Using the gauge condition, we can eliminate four of them, leaving six independent degrees of freedom. Unlike (23), the above solution does not admit any residual gauge transformation to further eliminate degrees of freedom. Any attempt to perform a residual gauge transformation would take the solution "off shell," i.e., the equations of motion would not be obeyed.

On the other hand, we have to ensure that $h_{\mu \nu} \ll 1$. However, in general this does not eliminate any degree of freedom; it is just a requirement of the linearized theory. This translates in requiring the first term in the expansion of the hyperbolic cosine to cancel the $-\eta_{\mu \nu}$ piece in (33), or in other words

$$
\int \frac{d^{4} k}{(2 \pi)^{4}} \delta\left(k^{2}-2 \Lambda\right) A(k)=4 .
$$

Since (33) is the most general solution to the equations, we must be able to recover the solutions in the previous section by performing an expansion in $\Lambda$. To do so, we only have to choose the right form for $E_{\mu \nu}(k), D_{\mu \nu}(k), A(k)$, 
and $B(k)$. As mentioned previously, to reach a Lorentzcovariant formulation such as (23) in the Lorenz gauge, we can safely assume that $D_{\mu \nu}$ and $B$ are zero as the resulting metric must satisfy $h_{\mu \nu}(x)=h_{\mu \nu}(-x)$, as discussed. In addition, $A(k)$ can only be a constant on Lorentz covariance grounds. We will take it to be $A(k) \equiv$ $\frac{A^{\prime}}{k^{2}}=\frac{A^{\prime}}{2 \Lambda}$. Also, $E_{\mu \nu}$ needs to be a (traceless) Lorentz- covariant tensor, namely $E_{\mu \nu}(k) \equiv \frac{E}{2 \Lambda}\left(k_{\mu} k_{\nu}-\frac{\eta_{\mu \nu}}{2} \Lambda\right)$. The proportionality coefficient between $E$ and $A^{\prime}$ comes from the gauge condition (34). Finally, as also indicated previously, the integrals require a finite support to be welldefined, and this should be implemented in a Lorentzinvariant way too; a sharp cutoff will be used below, although this is not crucial at all. Expanding (33),

$$
\begin{aligned}
h_{\mu \nu} & =\int \frac{d^{4} k}{(2 \pi)^{4}} \delta\left(k^{2}-2 \Lambda\right)\left(E_{\mu \nu}(k) \cos k x+\frac{\eta_{\mu \nu}}{4} A(k) \cosh k x\right)-\eta_{\mu \nu} \\
& =\int \frac{d^{4} k}{(2 \pi)^{4}} \delta\left(k^{2}-2 \Lambda\right)\left(E_{\mu \nu}(k)\left(1-\frac{(k \cdot x)^{2}}{2}+\ldots\right)+\frac{\eta_{\mu \nu}}{4} A(k)\left(1+\frac{(k \cdot x)^{2}}{2}+\ldots\right)\right)-\eta_{\mu \nu}
\end{aligned}
$$

and using the definitions given above,

$$
h_{\mu \nu} \simeq \int \frac{d^{3} \vec{k}}{(2 \pi)^{3}} \frac{1}{2 \sqrt{2 \Lambda+\vec{k}^{2}}}\left(\frac{E}{2 \Lambda}\left(k_{\mu} k_{\nu}-\frac{\eta_{\mu \nu}}{2} \Lambda\right)\left(1-\frac{(k \cdot x)^{2}}{2}\right)+\frac{\eta_{\mu \nu}}{4} \frac{A^{\prime}}{2 \Lambda}\left(1+\frac{(k \cdot x)^{2}}{2}\right)\right)-\eta_{\mu \nu} .
$$

Now we introduce the cutoff, $\sqrt{2 \Lambda}$. Already condition (36) dictates the value for $A^{\prime}=\frac{32 \pi^{2}}{C}$, where $C=\frac{1}{\Lambda} \int_{0}^{\sqrt{2 \Lambda}} d|\vec{k}| \frac{\vec{k}^{2}}{\sqrt{2 \Lambda+\vec{k}^{2}}}$. Then the solution reads

$$
\begin{aligned}
h_{\mu \nu} & \simeq \int_{0}^{\sqrt{2 \Lambda}} \frac{d|\vec{k}|}{2 \pi^{2}} \frac{\vec{k}^{2}}{2 \sqrt{2 \Lambda+\vec{k}^{2}}}\left(-\frac{E}{2 \Lambda}\left(k_{\mu} k_{\nu}-\frac{\eta_{\mu \nu}}{2} \Lambda\right) \frac{(k \cdot x)^{2}}{2}+\frac{\eta_{\mu \nu}}{4} \frac{16 \pi^{2}}{\Lambda C} \frac{(k \cdot x)^{2}}{2}\right) \\
& =\int_{0}^{\sqrt{2 \Lambda}} \frac{d|\vec{k}|}{2 \pi^{2}} \frac{\vec{k}^{2}}{2 \sqrt{2 \Lambda+\vec{k}^{2}}}\left(-E\left(\frac{\Lambda}{24}\left(\eta_{\mu \nu} x^{2}+2 x_{\mu} x_{\nu}\right)-\frac{\Lambda}{16} \eta_{\mu \nu} x^{2}\right)+\eta_{\mu \nu} x^{2} \frac{\pi^{2}}{C}\right) \\
& =\frac{\Lambda C}{4 \pi^{2}}\left(-E\left(\frac{\Lambda}{24}\left(\eta_{\mu \nu} x^{2}+2 x_{\mu} x_{\nu}\right)-\frac{\Lambda}{16} \eta_{\mu \nu} x^{2}\right)+\eta_{\mu \nu} x^{2} \frac{\pi^{2}}{C}\right) .
\end{aligned}
$$

The value of $E$ is fixed via the gauge condition (34) to $E=-\frac{16 \pi^{2}}{3 C \Lambda}$, leaving the perturbation in the form

$$
h_{\mu \nu} \simeq \frac{\Lambda}{9}\left(x_{\mu} x_{\nu}+2 \eta_{\mu \nu} x^{2}\right),
$$

which is precisely (23).

\section{WAVELIKE SOLUTIONS}

In this section, we will finally investigate the effects of the cosmological constant in the propagation of GW in the appropriate coordinate system.

\section{A. Lowest order solutions}

We write $h_{\mu \nu}=h_{\mu \nu}^{\Lambda}+h_{\mu \nu}^{W}$. The term $h_{\mu \nu}^{\Lambda}$ is the solution we just found; $h_{\mu \nu}^{W}$ will be a perturbation on the metric induced by some source of GW. The same decomposition holds for the trace-reversed metric $\tilde{h}_{\mu \nu}$. Waves are usually considered in the transverse traceless gauge [21]

$$
\tilde{h}_{\mu}^{W \mu}=h_{\mu}^{W \mu}=0, \quad \partial_{\mu} h_{\nu}^{W \mu}=\partial_{\mu} \tilde{h}_{\nu}^{W \mu}=0 .
$$

This is compatible with the $\Lambda$ gauge condition as the right-hand side of (11) is unchanged when considering $\tilde{h}_{\mu \nu}^{\Lambda}+\tilde{h}_{\mu \nu}^{W}$ provided that (11) is fulfilled by $h_{\mu \nu}^{\Lambda}$. This also makes clear that, at this order, the gauge condition involves the perturbation associated to the background and not the metric perturbation associated to a gravitational wave.

Since the proper equations of motion in the Lorenz gauge at this order, neglecting $\mathcal{O}\left(\Lambda h_{\mu \nu}\right)$, are just $\square h_{\mu \nu}=$ $h_{\mu \nu}^{\Lambda}+\square h_{\mu \nu}^{W}=0$, being the latter an independent perturbation, it is obvious that

$$
\square h_{\mu \nu}^{W}=0,
$$

and the gravitational wave solutions are in these coordinate systems functionally identical to those existing in flat space.

Note that because the $\Lambda h_{\mu \nu}^{\Lambda}$ has been neglected, the remaining residual gauge invariance allows for a removal of 4 of the 6 degrees of freedom in $h_{\mu \nu}^{W}$, and the analogy with wave propagation in Minkowski space is complete.

In the case of the lowest order equations, the full solution of (20) is 


$$
\begin{aligned}
h_{\mu \nu} & =h_{\mu \nu}^{\Lambda}+h_{\mu \nu}^{W} \\
& =\frac{\Lambda}{9}\left(x_{\mu} x_{\nu}+2 \eta_{\mu \nu} x^{2}\right)+E_{\mu \nu}^{W} \cos k x+D_{\mu \nu}^{W} \sin k x,
\end{aligned}
$$

where $E^{W}=D^{W}=0, k_{\mu} E_{\nu}^{\mu W}=k_{\mu} D_{\nu}^{\mu W}=0$, and $k^{2}=0$.

Now we want to see what plane waves such as the ones in (43) look like in the new coordinate system. Transformation (17) acts both on the polarization tensors and on the arguments of the sine and cosine. For the polarization tensors, we can always cut the expansion in $\Lambda$ and keep terms only up to a certain order. However, the transformation on the arguments yields terms of the type
$Z^{3} w \Lambda$, which in general can be relevant. The sine and cosine cannot be expanded; we have to transform the argument exactly. We shall later evaluate the error caused by retaining only the lowest order terms in the arguments.

For the polarization tensors, since we transform them independently of the arguments, it is easy to see qualitatively what the corrections to the polarization tensors will be. On dimensional grounds alone, all corrections will be of the order $\mathcal{O}(\sqrt{\Lambda} Z)$ or at most $\mathcal{O}\left(\Lambda Z^{2}\right)$, being that these quantities in the region of validity of the approximation are very small.

Nonetheless, the transformed wavelike solution to order $\sqrt{\Lambda}$ is

$$
\begin{aligned}
h_{\mu \nu}^{W_{\mathrm{FRW}}}= & \left(\begin{array}{cccc}
0 & 0 & 0 & 0 \\
0 & E_{11}\left(1+2 \sqrt{\frac{\Lambda}{3}} T\right) & E_{12}\left(1+2 \sqrt{\frac{\Lambda}{3}} T\right) & 0 \\
0 & E_{12}\left(1+2 \sqrt{\frac{\Lambda}{3}} T\right) & -E_{11}\left(1+2 \sqrt{\frac{\Lambda}{3}} T\right) & 0 \\
0 & 0 & 0 & 0
\end{array}\right) \times \cos \left(w(T-Z)+w \sqrt{\frac{\Lambda}{3}}\left(\frac{Z^{2}}{2}-T Z\right)+\mathcal{O}(\Lambda)\right)+\mathcal{O}(\Lambda) \\
& +\left(\begin{array}{cccc}
0 & 0 & 0 & 0 \\
0 & D_{11}\left(1+\sqrt{\frac{\Lambda}{3}} T\right) & D_{12}\left(1+\sqrt{\frac{\Lambda}{3}} T\right) & 0 \\
0 & D_{12}\left(1+\sqrt{\frac{\Lambda}{3}} T\right) & -D_{11}\left(1+\sqrt{\frac{\Lambda}{3}} T\right) & 0 \\
0 & 0 & 0 & 0
\end{array}\right) \times \sin \left(w(T-Z)+w \sqrt{\frac{\Lambda}{3}}\left(\frac{Z^{2}}{2}-T Z\right)+\mathcal{O}(\Lambda)\right)+\mathcal{O}(\Lambda) .
\end{aligned}
$$

The term $w(T-Z)$ dominates the argument of the trigonometric functions, and it can be checked numerically that the error made by omitting terms of order $\Lambda$ or higher is $\leqslant 10^{-3}$ for the purposes of the next section.

\section{B. Next-order solutions}

As we have argued before, it is not justified to neglect the term of order $\Lambda h_{\mu \nu}^{W}$ in this case. As unlike for the case of the background, the magnitude of the two quantities is unrelated. We can add a wavelike piece to the solution (33)

$$
\begin{aligned}
h_{\mu \nu} & =h_{\mu \nu}^{\Lambda}+h_{\mu \nu}^{W} \\
& =\int \frac{d^{4} k}{(2 \pi)^{4}} \delta\left(k^{2}-2 \Lambda\right)\left(E_{\mu \nu} \cos k x+D_{\mu \nu} \sin k x+\frac{\eta_{\mu \nu}}{4}(A \cosh k x+B \sinh k x)\right)-\eta_{\mu \nu}+E_{\mu \nu}^{W} \cos k x+D_{\mu \nu}^{W} \sin k x .
\end{aligned}
$$

This will always be a solution of (5) and (9) as long as $E^{W}=D^{W}=0, k_{\mu} E_{\nu}^{W \mu}=k_{\mu} D_{\nu}^{W \mu}=0$, and $k^{2}=2 \Lambda$. However, now we are not allowed to perform any gauge transformation, at least at the next-order level. We can still use the gauge condition and the traceless condition to eliminate 5 degrees of freedom from the wave. We are left with a massive wave with 5 degrees of freedom. The polarization vectors of which, for a wave propagating in the $z$ direction $\left(k_{1}=k_{2}=0\right)$, can be written as

$$
E_{\mu \nu}^{W}=\left(\begin{array}{cccc}
E_{00} & \frac{\sqrt{w^{2}-2 \Lambda}}{w} E_{13} & \frac{\sqrt{w^{2}-2 \Lambda}}{w} E_{23} & \frac{w}{\sqrt{w^{2}-2 \Lambda}} E_{00} \\
\frac{\sqrt{w^{2}-2 \Lambda}}{w} E_{13} & E_{11} & E_{12} & E_{13} \\
\frac{\sqrt{w^{2}-2 \Lambda}}{w} E_{23} & E_{12} & -E_{11}-E_{00} \frac{2 \Lambda}{w^{2}-2 \Lambda} & E_{23} \\
\frac{w}{\sqrt{w^{2}-2 \Lambda}} E_{00} & E_{13} & E_{23} & \frac{w^{2}}{w^{2}-2 \Lambda} E_{00}
\end{array}\right) .
$$


And there is a similar expression for $D_{\mu \nu}^{W}$. At the exact level, this is as far as one can go, but in order to understand the meaning of these massive waves, we turn again to an expansion in powers of $\Lambda$. We will proceed in two steps. First, we expand the solution in powers of $\Lambda$ and collect terms order by order. Then, using the same reasoning in the equations of motion, we can use an approximate residual invariance to rewrite the polarization tensors as the usual GW in Minkowski space-time plus an order $\Lambda$ contribution with the extra degrees of freedom.

The polarization vectors (46) can then be written as

$$
\begin{aligned}
E_{\mu \nu}^{W}= & \left(\begin{array}{llll}
E_{00} & E_{13} & E_{23} & E_{00} \\
E_{13} & E_{11} & E_{12} & E_{13} \\
E_{23} & E_{12} & -E_{11} & E_{23} \\
E_{00} & E_{13} & E_{23} & E_{00}
\end{array}\right) \\
& +\left(\begin{array}{cccc}
0 & -\frac{\Lambda}{w^{2}} E_{13} & -\frac{\Lambda}{w^{2}} E_{23} & \frac{\Lambda}{w^{2}} E_{00} \\
-\frac{\Lambda}{w^{2}} E_{13} & 0 & 0 & 0 \\
-\frac{\Lambda}{w^{2}} E_{23} & 0 & -E_{00} \frac{2 \Lambda}{w^{2}} & 0 \\
\frac{\Lambda}{w^{2}} E_{00} & 0 & 0 & \frac{2 \Lambda}{w^{2}} E_{00}
\end{array}\right)+\mathcal{O}\left(\Lambda^{2}\right) \\
& \equiv E_{\mu \nu}^{(0)}+E_{\mu \nu}^{(1)}+\mathcal{O}\left(\Lambda^{2}\right) .
\end{aligned}
$$

The same decomposition applies to $D_{\mu \nu}^{W}$. This expansion makes explicit the contributions of $\Lambda$ at a given order. We want to expand

$$
h_{\mu \nu}^{W}=h_{\mu \nu}^{(0)}+h_{\mu \nu}^{(1)}+\mathcal{O}\left(\Lambda^{2}\right),
$$

where the superscript refers to the order in $\Lambda$. The functions sine and cosine can also be expanded around a massless wave with coordinate-dependent amplitudes [15]

$$
\begin{aligned}
h_{\mu \nu}^{W}= & E_{\mu \nu}^{W} \cos k x+D_{\mu \nu}^{W} \sin k x \\
\simeq & {\left[\left(E_{\mu \nu}^{W}-\frac{\Lambda z}{w} D_{\mu \nu}^{W}\right) \cos w(t-z)\right.} \\
& \left.+\left(D_{\mu \nu}^{W}+\frac{\Lambda z}{w} E_{\mu \nu}^{W}\right) \sin w(t-z)\right]
\end{aligned}
$$

or what is tantamount,

$$
\begin{aligned}
h_{\mu \nu}^{W}= & {\left[\left(E_{\mu \nu}^{(0)}+E_{\mu \nu}^{(1)}-\frac{\Lambda z}{w} D_{\mu \nu}^{(0)}\right) \cos w(t-z)\right.} \\
& \left.+\left(D_{\mu \nu}^{(0)}+D_{\mu \nu}^{(1)}+\frac{\Lambda z}{w} E_{\mu \nu}^{(0)}\right) \sin w(t-z)\right]+\mathcal{O}\left(\Lambda^{2}\right) .
\end{aligned}
$$

We see that the massive wave we started with can be written at linear order in the cosmological constant in terms of a massless wave where all dependency in $\Lambda$ appears only through the polarization tensors

$h_{\mu \nu}^{W}=E_{\mu \nu}^{W} \cos w(t-z)+D_{\mu \nu}^{W} \sin w(t-z)+\mathcal{O}\left(\Lambda^{2}\right)$, where $E_{\mu \nu}^{W}$ and $D_{\mu \nu}^{W}$ can be read from (50). The above is a valid solution of $\square h_{\mu \nu}^{W}+2 \Lambda h_{\mu \nu}^{W}=0$ only to order $\Lambda$ (included), which means we can expand the equations of motion to the same order without loss of validity,

$$
\square h_{\mu \nu}^{(0)}+\square h_{\mu \nu}^{(1)}+2 \Lambda h_{\mu \nu}^{(0)}+\mathcal{O}\left(\Lambda^{2}\right)=0 .
$$

Now we can split the problem and solve order by order,

$$
\square h_{\mu \nu}^{(0)}=0 \quad \square h_{\mu \nu}^{(1)}+2 \Lambda h_{\mu \nu}^{(0)}=0 .
$$

Because of the fact that (52) is not exact, the solution to it can admit a residual gauge transformation that will take the solution off shell some order beyond the order we consider. The transformed solution is

$$
\square h_{\mu \nu}^{\prime(0)}=0 \quad \square h_{\mu \nu}^{(1)}+2 \Lambda h_{\mu \nu}^{(0)}=0 .
$$

The first equation in (54) is analogous to (42), i.e., residual transformations on $h_{\mu \nu}^{(0)}$ are not restricted. To order zero, we obtain GW analogous to the ones in flat space (in the present set of coordinates, that is). But, in this case, the transformation propagates to the following order through the second equation in (54), making it necessary to find the transformed $h_{\mu \nu}^{\prime(1)}$.

It is not difficult to see that the following polarization tensor fulfills the necessary requirements of tracelessness as well as the gauge condition $\left(k_{\mu} E_{\nu}^{W \mu}=k_{\mu} D_{\nu}^{W \mu}=0\right)$

$E_{\mu \nu}^{W}=\left(\begin{array}{cccc}\frac{\Lambda}{w^{2}} E_{00} & -\frac{\Lambda}{w^{2}} E_{13} & -\frac{\Lambda}{w^{2}} E_{23} & \frac{\Lambda}{w^{2}} E_{00} \\ -\frac{\Lambda}{w^{2}} E_{13} & E_{11}-\frac{\Lambda z}{w} D_{11} & E_{12}-\frac{\Lambda z}{w} D_{12} & -\frac{\Lambda}{w^{2}} E_{13} \\ -\frac{\Lambda}{w^{2}} E_{23} & E_{12}-\frac{\Lambda z}{w} D_{12} & -E_{11}+\frac{\Lambda z}{w} D_{11} & -\frac{\Lambda}{w^{2}} E_{23} \\ \frac{\Lambda}{w^{2}} E_{00} & -\frac{\Lambda}{w^{2}} E_{13} & -\frac{\Lambda}{w^{2}} E_{23} & \frac{\Lambda}{w^{2}} E_{00}\end{array}\right)$.

$D_{\mu \nu}$ is similarly obtained from (50). Notice the presence of the usual components (of $\mathcal{O}(1)$ ) in the polarization tensor in the $x, y$ entries of the metric.

To this order in $\Lambda$, we obtain massless waves with coordinate-dependent modified amplitudes, which depend on $\Lambda$. We can see that the extra degrees of freedom due to the form of the linearized equations of motion for nonzero $\Lambda$ will only couple to matter fields proportionally to $\Lambda$ thanks to the coupling $h_{\mu \nu}^{W} T^{\mu \nu}$ and thus will be irrelevant in practice.

\section{Transformed next-order solutions}

Now we are ready to apply the series of coordinate transformations (27), (29), (31), and (17) to the wavelike solution (51) that we found in the previous subsection in order to obtain a physical expression in FRW coordinates. Recall the waves in the general Lorenz gauge read

$$
h_{\mu \nu}^{W}=E_{\mu \nu}^{W}(\Lambda, z) \cos w(t-z)+D_{\mu \nu}^{W}(\Lambda, z) \sin w(t-z),
$$


where $E_{\mu \nu}^{W}$ can be read off from (55). From (56), it is clear the only modification with respect to the plane waves of the lower order is in the polarization tensors, being already of order $\Lambda$. This suggests that all the new modifications to order $\Lambda$ of the next-order waves are due to the change of coordinates. Explicitly, the transformed waves to order $\Lambda$ read

$$
\begin{aligned}
& h_{\mu \nu}^{W_{\mathrm{RRW}}}\left[\begin{array}{cccc}
\frac{\Lambda}{w^{2}} E_{00} & -\frac{\Lambda}{w^{2}} E_{13} & -\frac{\Lambda}{w^{2}} E_{23} & \frac{\Lambda}{w^{2}} E_{00} \\
-\frac{\Lambda}{w^{2}} E_{13} & E_{11}-\frac{\Lambda Z}{w} D_{11} & E_{12}-\frac{\Lambda Z}{w} D_{12} & -\frac{\Lambda}{w^{2}} E_{13} \\
-\frac{\Lambda}{w^{2}} E_{23} & E_{12}-\frac{\Lambda Z}{w} D_{12} & -E_{11}+\frac{\Lambda Z}{w} D_{11} & -\frac{\Lambda}{w^{2}} E_{23} \\
\frac{\Lambda}{w^{2}} E_{00} & -\frac{\Lambda}{w^{2}} E_{13} & -\frac{\Lambda}{w^{2}} E_{23} & \frac{\Lambda}{w^{2}} E_{00}
\end{array}\right) \\
& \left.+\left(\begin{array}{cccc}
0 & E_{11}\left(2 \sqrt{\frac{\Lambda}{3}} T+\frac{2 \Lambda}{9} T^{2}+\frac{5 \Lambda}{18} Z^{2}\right) & E_{12}\left(2 \sqrt{\frac{\Lambda}{3}} T+\frac{2 \Lambda}{9} T^{2}+\frac{5 \Lambda}{18} Z^{2}\right) & 0 \\
0 & E_{12}\left(2 \sqrt{\frac{\Lambda}{3}} T+\frac{2 \Lambda}{9} T^{2}+\frac{5 \Lambda}{18} Z^{2}\right) & -E_{11}\left(2 \sqrt{\frac{\Lambda}{3}} T+\frac{2 \Lambda}{9} T^{2}+\frac{5 \Lambda}{18} Z^{2}\right) & 0 \\
0 & 0 & 0 & 0
\end{array}\right)+\mathcal{O}\left(\Lambda^{3 / 2}\right)\right] \\
& \times \cos \left(w(T-Z)+w \sqrt{\frac{\Lambda}{3}}\left(\frac{Z^{2}}{2}-T Z\right)-\frac{1}{18} w \Lambda\left(T^{3}+T^{2} Z-5 T Z^{2}+2 Z^{3}\right)+\mathcal{O}\left(\Lambda^{3 / 2}\right)\right) \\
& +\left[\begin{array}{cccc}
\frac{\Lambda}{w^{2}} D_{00} & -\frac{\Lambda}{w^{2}} D_{13} & -\frac{\Lambda}{w^{2}} D_{23} & \frac{\Lambda}{w^{2}} D_{00} \\
-\frac{\Lambda}{w^{2}} D_{13} & D_{11}+\frac{\Lambda Z}{w} E_{11} & D_{12}+\frac{\Lambda Z}{w} E_{12} & -\frac{\Lambda}{w^{2}} D_{13} \\
-\frac{\Lambda}{w^{2}} D_{23} & D_{12}+\frac{\Lambda Z}{w} E_{12} & -D_{11}-\frac{\Lambda Z}{w} E_{11} & -\frac{\Lambda}{w^{2}} D_{23} \\
\frac{\Lambda}{w^{2}} D_{00} & -\frac{\Lambda}{w^{2}} D_{13} & -\frac{\Lambda}{w^{2}} D_{23} & \frac{\Lambda}{w^{2}} D_{00}
\end{array}\right) \\
& \left.+\left(\begin{array}{cccc}
0 & 0 & 0 & 0 \\
0 & D_{11}\left(2 \sqrt{\frac{\Lambda}{3}} T+\frac{2 \Lambda}{9} T^{2}+\frac{5 \Lambda}{18} Z^{2}\right) & D_{12}\left(2 \sqrt{\frac{\Lambda}{3}} T+\frac{2 \Lambda}{9} T^{2}+\frac{5 \Lambda}{18} Z^{2}\right) & 0 \\
0 & D_{12}\left(2 \sqrt{\frac{\Lambda}{3}} T+\frac{2 \Lambda}{9} T^{2}+\frac{5 \Lambda}{18} Z^{2}\right) & -D_{11}\left(2 \sqrt{\frac{\Lambda}{3}} T+\frac{2 \Lambda}{9} T^{2}+\frac{5 \Lambda}{18} Z^{2}\right) & 0 \\
0 & 0 & 0 & 0
\end{array}\right)+\mathcal{O}\left(\Lambda^{3 / 2}\right)\right] \\
& \times \sin \left(w(T-Z)+w \sqrt{\frac{\Lambda}{3}}\left(\frac{Z^{2}}{2}-T Z\right)-\frac{1}{18} w \Lambda\left(T^{3}+T^{2} Z-5 T Z^{2}+2 Z^{3}\right)+\mathcal{O}\left(\Lambda^{3 / 2}\right)\right) .
\end{aligned}
$$

\section{DETECTABILITY}

Let us now do some order-of magnitude estimates to evaluate the effect of the corrections induced by $\Lambda \neq 0$ on the propagation of gravitational waves.

For the polarization tensors, we have not attempted to derive the $\Lambda$-order corrections in full detail, although this is possible, because already the most relevant correction, i.e., $\sqrt{\Lambda} Z E_{\mu \nu}^{(0)}$, has to be some orders of magnitude smaller than $E_{\mu \nu}^{(0)}$ for the approximation to be valid. For example, for a coordinate value of the order of a typical distance to a supernova,
$10^{23} \mathrm{~m}$, the quantity $\sqrt{\Lambda} Z \sim 10^{-3}\left(\Lambda \sim 10^{-52} \mathrm{~m}^{-2} \sim\right.$ $10^{-35} \mathrm{~s}^{-2}$ ). This already means a small correction to an amplitude that has so far escaped detection and which presumably will not be measured with sufficient precision to discern the effect of the $\Lambda$-order effects in the foreseeable future. However, conceptually it is an interesting result.

It is more interesting to work out the corrections to the dispersion relation for (44). As previously, let us consider waves that propagate in the $Z$ direction and are monochromatic. The maxima of the wave will be reached when 


$$
w(T-Z)+w \sqrt{\frac{\Lambda}{3}}\left(\frac{Z^{2}}{2}-T Z\right)=n \pi,
$$

or

$$
Z_{\text {max }}(n, T)=T-\frac{n \pi}{w}-\frac{T^{2}}{2} \sqrt{\frac{\Lambda}{3}}+\frac{n^{2} \pi^{2}}{2 w^{2}} \sqrt{\frac{\Lambda}{3}} .
$$

From (59), we can also calculate the phase velocity of the wave which is defined as

$$
v_{p}(T) \equiv \frac{d Z_{\max }}{d T}=1-T \sqrt{\frac{\Lambda}{3}}+\mathcal{O}(\Lambda) .
$$

We see that in comoving coordinates the phase velocity is smaller than 1. This does not mean that the waves slow down. We can calculate the velocity in "ruler" distance. For a fixed time, we have

$$
\begin{aligned}
-d l^{2} & =-\left(1+T \sqrt{\frac{\Lambda}{3}}\right) d Z^{2} \\
\frac{d l}{d T} & =\frac{d}{d T}\left[\left(1+T \sqrt{\frac{\Lambda}{3}}\right) d Z_{\max }\right]=1 .
\end{aligned}
$$

It is also interesting to rewrite the trigonometric functions of the wave defining $w_{\text {eff }}(Z) \equiv w\left(1-Z \sqrt{\frac{\Lambda}{3}}\right)$

$$
\begin{aligned}
& \cos \left[T w\left(1-Z \sqrt{\frac{\Lambda}{3}}\right)-Z w\left(1-Z \sqrt{\frac{\Lambda}{3}}\right)\right] \\
& =\cos w_{\mathrm{eff}}(T-Z) .
\end{aligned}
$$

Note that the transformed wave corresponds to a usual wave with an effective frequency dependent on the coordinate $Z$. The wave becomes redshifted as it propagates away from the source.

To see explicitly the effect of $\Lambda$ in the propagation of a wave described in comoving coordinates, we plot (Fig. 1) one of the $h_{++}$components of the wave for a given instant ( $T=0$ for simplicity). A wave with a physical frequency ranging $10^{3} \mathrm{~Hz}<w<10^{-10} \mathrm{~Hz}$ cannot be practically plotted in the relevant $Z$ range. To see the effect in a few cycles, we take $w=4 \cdot 10^{-16} \mathrm{~Hz}$, which does not affect the overall magnitude of the correction. We plot the wave for $\Lambda=10^{-52} \mathrm{~m}^{-2}$ and for $\Lambda=10^{-51} \mathrm{~m}^{-2}$ to assess the influence of $\Lambda$ on the wave propagation. Then we plot $h_{++} \sim\left(1+\frac{5}{9} \Lambda Z^{2}\right) \cos \left[-Z w\left(1-Z \sqrt{\frac{\Lambda}{3}}\right)\right]$.

From these results, we can already draw some conclusions. The genuine corrections due to the masslike term in (9) remain unchanged in the transformed waves if we cut the expansion to order $\mathcal{O}(\Lambda)$. Moreover, they are of order $\frac{\Lambda Z}{w}$, which is in practice irrelevant unless the value of $\Lambda$ is much greater than the current value. However, transformation (17) induces modifications to the wave, both in the

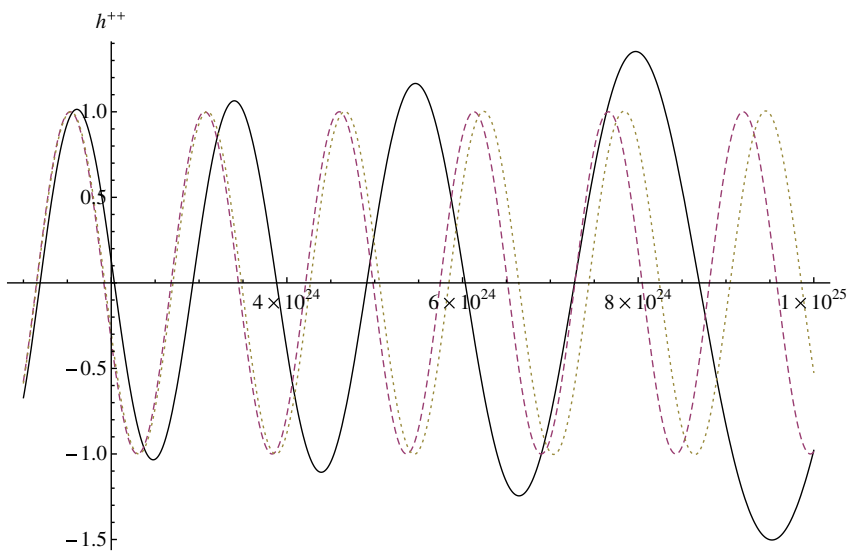

FIG. 1 (color online). Dependency of the amplitude and wavelength on the coordinate distance $Z$ (expressed in meters) for a constant value of $T$ and for different values of $\Lambda$ : The dashed line corresponds to $\Lambda=0$, the dotted line corresponds to $\Lambda=10^{-52} \mathrm{~m}^{-2}$, and the solid line corresponds to $\Lambda=10^{-51} \mathrm{~m}^{-2}$.

amplitude and the phase, of order $\sqrt{\Lambda}$ and $\Lambda$. These modifications result in a simultaneous increase of the wavelength and of the amplitude with the coordinate $Z$. As shown in Fig. 1, the most interesting region for detection would be that of events (supernovae and pulsars, for example) happening at a distance $Z \sim 10^{23}-10^{25} \mathrm{~m}$ away, for which the correction $\sqrt{\frac{\Lambda}{3}} Z \sim 10^{-1}-10^{-3}$ is not negligible and is well within the validity range of the approximation. In fact, to take this type of correction into account seems probably essential to properly accounting for the measurements of this type of phenomena in pulsar arrays.

\section{SUMMARY}

The purpose of this work was to investigate the effect of the cosmological constant in the propagation of gravitational waves in a linearized theory of gravity. The presence of $\Lambda$ leads unavoidably to the curvature of the background space-time in which the waves propagate. Within the linearized approximation, in which the wave description corresponds to perturbation solutions of an harmonic wave equation, this leads to a decomposition $g_{\mu \nu} \simeq \eta_{\mu \nu}+h_{\mu \nu}^{\Lambda}+h_{\mu \nu}^{W}$, including a modification of the background (corresponding to the curvature) and a wavelike perturbation.

To see the way the propagation of the waves is affected, first one has to understand the implications that the different coordinate choices (gauge choices) have in the resolution of the equations of motion as well as the importance of the terms of different order retained in the linearization. One is free to choose any particular gauge to solve the equations, however, since the linearized Einstein equations are not invariant under general coordinate transformations, 
their form will depend on the gauge choice. We argue that the above procedure of linearization is consistent in some coordinate systems but not in others. In particular, it is inconsistent to linearize the equations in the familiar Friedmann-Robertson-Walker cosmological coordinates (the metric only depends on time).

Einstein equations can, however, be consistently linearized in Schwarzschild-de Sitter coordinates; then $h_{\mu \nu}^{\Lambda}$ corresponds to a linearized version of the $\mathrm{SdS}$ metric, expanded to the first order in $\Lambda$. This metric can be easily modified to fulfill the Lorenz gauge condition. In this particular gauge, i.e., in this particular choice of coordinates, the analysis of gravitational waves follows a pattern very similar to the one in Minkowski space-time. In the case where the $\Lambda h_{\mu \nu}$ term is dropped, the residual gauge freedom of the Lorenz gauge allows for the removal of 4 additional degrees of freedom in the general solution, leaving the wavelike component with the usual 2 physical degrees of freedom of waves propagating in flat spacetime.

On the contrary, if the term $\Lambda h_{\mu \nu}$ is retained in the equations of motion, the situation changes. Even in the Lorenz gauge, the invariance under residual gauge transformations is lost. Again, it is not hard to find the most general solution to the linearized equations composed of a background and wavelike components. We prove the background solution to be consistent with the result previously found if $\Lambda$ is small. Since there is no residual invariance, the wavelike solution has to be interpreted as a massive wave with 5 degrees of freedom (the gauge condition and the trace condition amount to five constraints). However, we can make use of the approximate residual invariance at the leading order in $\Lambda$ to rewrite the solution as massless gravitational waves with position-dependent modified amplitudes that change very slowly given the current values of $\Lambda$. There are only two $\mathcal{O}(1)$ polarizations; the remaining degrees of freedom (up to the five independent ones required for a massive spin two wave) are of $\mathcal{O}(\Lambda)$ and couple extremely weakly to matter sources.

Finally, one has to transform these solutions to the physically significant FRW coordinates in order to extract observable consequences. At this point, modifications of $\mathcal{O}(\sqrt{\Lambda})$ appear. Numerically, these can be quite relevant for certain gravitational waves traveling from far away sources, and the effect of $\Lambda$ can absolutely have a detectable impact on pulsar timing arrays. Waves are modified both in the phase and the amplitude; in cosmological coordinates, they are redshifted in a prescribed way, and the amplitude of plane waves grows as they move away from the source.

\section{ACKNOWLEDGMENTS}

We acknowledge the financial support from the RTN ENRAGE and the research Grants No. FPA2007-66665, FPA2008-02878, FPA2010-20807, PROMETEO2008004, and SGR2009SGR502. This research is supported by the Consolider CPAN project. J.B. and D. E. would like to thank the CERN PH-TH Unit, where this research was initiated, for the hospitality extended to them. We thank J. Garcia-Bellido and R. Lapiedra for discussions on the subject.
[1] A. G. Riess et al. (Supernova Search Team Collaboration), Astron. J. 116, 1009 (1998); S. Perlmutter et al. (Supernova Cosmology Project Collaboration), Astrophys. J. 517, 565 (1999); A. G. Riess, Publ. Astron. Soc. Pac. 112, 1284 (2000); E. Linder and S. Perlmutter, Phys. World 20N12, 24 (2007); M. Kowalski et al. (Supernova Cosmology Project Collaboration), Astrophys. J. 686, 749 (2008); W. M. Wood-Vasey et al. (ESSENCE Collaboration), Astrophys. J. 666, 694 (2007); A. G. Riess and M. Livio, Astrophys. J. 648, 884 (2006); A. G. Riess et al. (Supernova Search Team Collaboration), Astrophys. J. 607, 665 (2004); D. N. Spergel et al. (WMAP Collaboration), Astrophys. J. Suppl. Ser. 170, 377 (2007); D. J. Eisenstein et al. (SDSS Collaboration), Astrophys. J. 633, 560 (2005); H. J. Seo and D. J. Eisenstein, Astrophys. J. 598, 720 (2003) and references therein; L. Fu et al., Astron. Astrophys. 479, 9 (2008); L. Guzzo et al., Nature (London) 451, 541 (2008).

[2] M. Sereno and P. Jetzer, Phys. Rev. D 73, 063004 (2006); A. Balaguera-Antolinez, C. G. Boehmer, and M. Nowakowski, Classical Quantum Gravity 23, 485 (2006);
L. Iorio, Adv. Astron. Astrophys., doi:10.1155/2008/ 268647 (2008); New Astron. Rev. 14, 196 (2009); Y. Suto, Prog. Theor. Phys. 90, 1173 (1993); arXiv:astroph/9609014; P. J. Peebles, Astrophys. Space Sci. 45, 3 (1976); The Large Scale Structure of the Universe (Princeton University, Princeton, NJ, 1980); G.S. Adkins, J. McDonnell, and R. N. Fell, Phys. Rev. D 75, 064011 (2007); See, for instance, W. Rindler, Relativity: Special, General, and Cosmological (Oxford University, New York, 2006), 2nd ed., ISBN 978-0-19-856731-8; , Phys. Lett. A 245, 363 (1998); the spherically-symmetric Schwarzschild-de Sitter solution was first discussed in F. Kottler, Ann. Phys. (Leipzig) 56, 420 (1918).

[3] J. Bernabeu, C. Espinoza, and N. E. Mavromatos, Phys. Rev. D 81, 084002 (2010).

[4] I. B. Khriplovich and A. A. Pomeransky, Int. J. Mod. Phys. D 17, 2255 (2008).

[5] M. Sereno, Phys. Rev. Lett. 102, 021301 (2009); Phys. Rev. D 77, 043004 (2008).

[6] W. Rindler and M. Ishak, Phys. Rev. D 76, 043006 (2007); M. Ishak, W. Rindler, J. Dossett, J. Moldenhauer, and 
C. Allison, Mon. Not. R. Astron. Soc. 388, 1279 (2008); M. Ishak and W. Rindler, Gen. Relativ. Gravit. 42, 2247 (2010).

[7] J. Bernabeu, C. Espinoza, N.E. Mavromatos, and V. Mitsou (unpublished).

[8] See, for instance, W. Rindler, Relativity: Special, General, and Cosmological (Oxford University, New York, 2006), 2nd ed., ISBN 978-0-19-856731-8; J. H. Taylor, Rev. Mod. Phys. 66, 711 (1994); J. M. Weisberg and J.H. Taylor, Astron. Soc. Pac. Conf. Ser. 328, 25 (2005).

[9] J. Abadie et al. (LIGO Collaboration), Nucl. Instrum. Methods Phys. Res., Sect. A 624, 223 (2010).

[10] J. Baker et al., Report No. LISA-LIST-RP-436, version $1.0,2007$.

[11] G. Hobs et al., Classical Quantum Gravity 27, 8 (2010).

[12] C. Cavilli and S. Rawlingd, in Science with the SKA, New Astronomy Reviews Vol. 48 (Elsevier, New York, 2004).

[13] See, for instance, H. Colins, Gravity's Shadow: The Search for Gravitational Waves (University of Chicago, Chicago, 2004).
[14] J. Bicak and J. Podolsky, J. Math. Phys. (N.Y.) 40, 4506 (1999).

[15] J. Näf, P. Jetzer, and M. Sereno, Phys. Rev. D 79, 024014 (2009).

[16] J. Bicak, J. Katz, and D. Lynden-Bell, Phys. Rev. D 76, 063501 (2007).

[17] D. Baumann, P. J. Steinhardt, K. Takahashi, and K. Ichiki, Phys. Rev. D 76, 084019 (2007).

[18] Lu Hui-qing, Chinese astronomy and astrophysics 12, 186 (1988).

[19] A. H. Guth, Phys. Rev. D 23, 347 (1981); A. D. Linde, Phys. Lett. 108B, 389 (1982); A. Albrecht and P. J. Steinhardt, Phys. Rev. Lett. 48, 1220 (1982).

[20] W.B. Bonnor, in Recent Developments in General Relativity: A Collection of Papers Dedicated to Leopold Infeld, edited by P. G. Bergmann (Pergamon, New York, 1962); W. Rindler, Phys. Lett. A 245, 363 (1998).

[21] See, e.g., R. H. Price and Y. Wang, Am. J. Phys. 76, 930 (2008). 